\title{
Lenalidomide after Stem-Cell Transplantation for Multiple Myeloma
}

\author{
Philip L. McCarthy, M.D., Kouros Owzar, Ph.D., Craig C. Hofmeister, M.D., David D. Hurd, \\ M.D., Hani Hassoun, M.D., Paul G. Richardson, M.D., Sergio Giralt, M.D., Edward A. \\ Stadtmauer, M.D., Daniel J. Weisdorf, M.D., Ravi Vij, M.D., Jan S. Moreb, M.D., Natalie Scott \\ Callander, M.D., Koen Van Besien, M.D., Teresa Gentile, M.D., Ph.D., Luis Isola, M.D., \\ Richard T. Maziarz, M.D., Don A. Gabriel, M.D., Ph.D., Asad Bashey, M.D., Ph.D., Heather \\ Landau, M.D., Thomas Martin, M.D., Muzaffar H. Qazilbash, M.D., Denise Levitan, M.D., \\ Brian McClune, M.D., Robert Schlossman, M.D., Vera Hars, M.S., John Postiglione, B.A., \\ Chen Jiang, Ph.D., Elizabeth Bennett, B.H.E., Susan Barry, B.A., Linda Bressler, Pharm.D., \\ Michael Kelly, M.A., Michele Seiler, M.S., Cara Rosenbaum, M.D., Parameswaran Hari, M.D., \\ Marcelo C. Pasquini, M.D., Mary M. Horowitz, M.D., Thomas C. Shea, M.D., Steven M. \\ Devine, M.D., Kenneth C. Anderson, M.D., and Charles Linker, M.D.
}

\section{Abstract}

BACKGROUND—Data are lacking on whether lenalidomide maintenance therapy prolongs the time to disease progression after autologous hematopoietic stem-cell transplantation in patients with multiple myeloma.

\begin{abstract}
METHODS-Between April 2005 and July 2009, we randomly assigned 460 patients who were younger than 71 years of age and had stable disease or a marginal, partial, or complete response 100 days after undergoing stem-cell transplantation to lenalidomide or placebo, which was administered until disease progression. The starting dose of lenalidomide was $10 \mathrm{mg}$ per day (range, 5 to 15 ).
\end{abstract}

\begin{abstract}
RESULTS-The study-drug assignments were unblinded in 2009, when a planned interim analysis showed a significantly longer time to disease progression in the lenalidomide group. At unblinding, $20 \%$ of patients who received lenalidomide and $44 \%$ of patients who received placebo had progressive disease or had died $(\mathrm{P}<0.001)$; of the remaining 128 patients who received placebo and who did not have progressive disease, 86 crossed over to lenalidomide. At a median follow-up of 34 months, 86 of 231 patients who received lenalidomide (37\%) and 132 of 229 patients who received placebo (58\%) had disease progression or had died. The median time to progression was 46 months in the lenalidomide group and 27 months in the placebo group $(\mathrm{P}<0.001)$. A total of 35 patients who received lenalidomide (15\%) and 53 patients who received placebo $(23 \%)$ died $(\mathrm{P}=0.03)$. More grade 3 or 4 hematologic adverse events and grade 3 nonhematologic adverse events occurred in patients who received lenalidomide $(\mathrm{P}<0.001$ for both comparisons). Second primary cancers occurred in 18 patients who received lenalidomide (8\%) and 6 patients who received placebo (3\%).
\end{abstract}

Copyright (@) 2012 Massachusetts Medical Society.

Address reprint requests to Dr. McCarthy at the BMT Program, Roswell Park Cancer Institute, Buffalo, NY 14263, or at philip.mccarthy@ roswellpark.org.

Drs. Anderson and Linker contributed equally to this article.

The authors' affiliations are listed in the Appendix.

Disclosure forms provided by the authors are available with the full text of this article at NEJM.org. 
CONCLUSIONS—Lenalidomide maintenance therapy, initiated at day 100 after hematopoietic stem-cell transplantation, was associated with more toxicity and second cancers but a significantly longer time to disease progression and significantly improved overall survival among patients with myeloma. (Funded by the National Cancer Institute; ClinicalTrials.gov number, NCT00114101.)

A goal of therapy for multiple myeloma, to induce complete remission and prolong survival, is usually accomplished with combination therapy. ${ }^{1,2}$ Autologous hematopoietic stem-cell transplantation is often used after induction chemotherapy to improve the response or to consolidate complete remission. ${ }^{1,2}$ However, since most patients with multiple myeloma have disease recurrence or progression after transplantation, maintenance therapies have been used to prolong complete remission and prevent relapse or progressive disease. Lowdose melphalan, interferon alfa, and glucocorticoids have been used for maintenance after primary therapy, but their long-term use is limited by toxicity and modest efficacy. ${ }^{3-6}$

Five studies involving patients who had undergone autologous hematopoietic stem-cell transplantation showed that thalidomide maintenance therapy improved progression-free survival, and three of the five studies showed improved overall survival. ${ }^{7-11}$ However, longterm thalidomide use is limited by toxicity. A sixth study showed no benefit with respect to progression-free survival or overall survival, but $77 \%$ of the patients did not complete maintenance therapy. ${ }^{12}$ Lenalidomide (Revlimid, Celgene), an orally administered, immunemodulating drug, has several mechanisms of action against multiple myeloma. ${ }^{13}$ It is an appealing agent for long-term use because of its activity when used alone at doses lower than induction doses and its favorable toxicity profile. ${ }^{14} \mathrm{We}$ designed a phase 3 , randomized, double-blind, placebo-controlled trial to determine whether lenalidomide therapy would prolong the time to disease progression in patients with multiple myeloma who had undergone induction therapy and a single stem-cell transplantation. Secondary end points included overall survival, the response after transplantation, and the feasibility of long-term administration.

\section{METHODS \\ PATIENTS}

Patients were eligible to participate in the trial if they had multiple myeloma and were 18 to 70 years of age. Other criteria were an Eastern Cooperative Oncology Group performance status ${ }^{15}$ of 0 or 1 (on a scale of 0 to 5 , with 0 indicating that the patient is fully active and 1 indicating that the patient is restricted in physically strenuous activity but ambulatory and able to carry out work of a light or sedentary nature), symptomatic disease requiring treatment (Durie-Salmon stage $\triangle 1$ ) (for definitions of the stages, see the Supplementary Appendix, available with the full text of this article at NEJM .org), and any induction regimen of 2 to 12 months' duration. At most, two induction regimens (excluding dexamethasone alone) could have been received.

Patients with stable disease or a marginal, partial, or complete response in the first 100 days after stem-cell transplantation were eligible. The minimum number of peripheral-blood stem cells (CD34+ cells) for transplantation was $2 \times 10^{6}$ per kilogram of body weight. Initially, the protocol mandated peripheral-blood stem-cell mobilization with cyclophosphamide and granulocyte colony-stimulating factor within 42 days before transplantation, but the protocol was subsequently amended to allow any mobilization and collection at any time before transplantation. Adequate pulmonary, cardiac, renal, and hepatic function was required, and all patients were registered before transplantation. Serious coexisting conditions, including uncontrolled diabetes, serious infections, and immune dysfunction, were exclusion criteria; pregnancy was also an exclusion criterion, and patients participated in an informational program regarding the unknown teratogenic potential of lenalidomide. 
After disease restaging, patients were randomly assigned in a blinded manner to lenalidomide or placebo between day 100 and day 110 after transplantation. All patients provided written informed consent. The study was approved by the Cancer and Leukemia Group B (CALGB), the Cancer Therapy Evaluation Program of the National Cancer Institute (NCI), and the NCI central institutional review board.

\section{STUDY TREATMENT AND OVERSIGHT}

The dosing schedule and adjustments, as well as guidelines for anticoagulation, are described in the Supplementary Appendix. The full protocol, along with the statistical analysis plan, is available at NEJM.org.

The NCI sponsored the study. Celgene provided the lenalidomide and placebo to the NCI, which in turn provided the study drugs to the investigators. Celgene had no involvement in the study design or conduct of the study or in the analysis or reporting of the data. The study principal investigator and the members of the statistical center vouch for the accuracy and completeness of the analysis and the data as reported by the site investigators. The site investigators agreed to participate in this study as members of their respective cooperative groups and are responsible for the completeness of data reporting and the fidelity of the study to the protocol.

\section{END POINTS AND DEFINITIONS}

The primary end point was time to progression, defined as time to progressive disease or death from any cause after transplantation. Response and progression were defined initially according to the criteria of the European Blood and Marrow Transplant Group ${ }^{16}$; these definitions were subsequently changed to be consistent with the criteria of the International Myeloma Working Group ${ }^{17}$ (see the Supplementary Appendix for details).

\section{STATISTICAL ANALYSIS}

The primary statistical hypothesis was that the time to progression was stochastically longer in the lenalidomide group than in the placebo group. The study was designed to have $90 \%$ power, with the use of the log-rank test at a one-sided significance level of 0.05 , to detect a hazard ratio of 1.4, assuming proportional hazards and an exponential time-to-event distribution. Under the assumed framework, 309 events were expected. The expected dropout rate before randomization was $15 \%$. Of 568 patients registered from 47 centers, 460 were randomly assigned to a study group with the use of a permuted-block design, stratified according to three baseline factors: normal or elevated serum $\beta_{2}$-microglobulin level at registration ( $\mathcal{S} .5 \mathrm{mg}$ per liter vs. $>2.5 \mathrm{mg}$ per liter $[\mathcal{S} 11.9 \mathrm{nmol}$ per liter vs. $>211.9 \mathrm{nmol}$ per liter]), prior use or nonuse of thalidomide during induction therapy; and prior use or nonuse of lenalidomide during induction therapy. The time-to-progression end point was monitored with the use of a group-sequential design for superiority and futility. Interim analyses of time to progression, overall survival, and adverse events were presented to the data and safety monitoring board of the CALGB twice a year when more than $20 \%$ of the expected events had occurred.

The data were released to the study team on December 17, 2009, after the third review because statistical evidence favored the lenalidomide group; this finding was observed after the first report to the data and safety monitoring board in June 2009. The analyses were based on the intention-to-treat principle and included follow-up data submitted on or before December 17, 2009 (the unblinding date) or follow-up data submitted as of October 31, 2011 (for evaluation of long-term outcomes). To assess the occurrence of second primary cancers reported after randomization, the nonprotocol end point of event-free survival, defined as time to first event (second primary cancer, progressive disease, or death) was 
considered. Starting in December 2010, the statistical center sent three sets of queries to all participating sites, and specific questionnaires were sent to all centers regarding cancer screening and second primary cancers. The last screening was conducted on January 1 , 2012, and no new cases had been reported since the October 31, 2011, data analysis.

Survival functions were estimated with the use of the Kaplan-Meier method. ${ }^{18}$

Discrepancies between survival functions were estimated with the use of the hazard ratios from a Cox model, ${ }^{19}$ under the implicit assumption of proportional hazards. To assess the predictive value of baseline covariables, a two-way multiplicative Cox model ${ }^{19}$ was used. To assess cause-specific risk (progression, death, and second primary cancers), the cumulative incidence curves were estimated with the use of the Kaplan-Meier method ${ }^{20}$ and compared with the use of the log-rank test proposed by Gray. ${ }^{21}$ All analyses were rightcensored since not all events had occurred at the time of the analysis, and as specified by the protocol, the date of transplantation was used as the reference date. The differences between proportions of patients with adverse events were tested with the use of Fisher's exact test ${ }^{22}$ and estimated with the use of a conditional maximum-likelihood estimator of the odds ratio. ${ }^{22}$ Asymptotic $\mathrm{P}$ values of less than $10^{-3}$ were denoted as $\mathrm{P}<0.001$. The analyses were conducted with R Statistical Environment software, version 2.14.1 (R Development Core Team 2011) along with survival and cmprsk extension packages. A detailed description of statistical considerations, including methods of design and analysis, is available in the Supplementary Appendix.

\section{RESULTS}

\section{CHARACTERISTICS OF THE PATIENTS}

Of the 568 patients enrolled in the study, 460 were randomly assigned to a study group: 231 to the lenalidomide group and 229 to the placebo group. (Fig. S1a and S1b in the Supplementary Appendix provide detailed information on the numbers of patients who were enrolled, assigned to a study group, and included in follow-up.) Age, sex, disease stage, and serum $\beta_{2}$-microglobulin level at registration were evenly distributed in the two groups (Table 1). Cytogenetic analysis was not required. The majority of patients received induction therapy with a regimen containing lenalidomide, thalidomide, or bortezomib, or a combination of the three (Table 1).

\section{TIME TO PROGRESSION AND OVERALL SURVIVAL}

The study was unblinded on December 17, 2009, after a median follow-up of 18 months, when 47 of the 231 patients in the lenalidomide group (20\%) as compared with 101 of the 229 patients in the placebo group $(44 \%)$ had progressive disease or had died $(\mathrm{P}<0.001)$. The hazard ratio for the risk of progression or death from any cause was 0.37 (95\% confidence interval [CI], 0.26 to 0.53 ), indicating a $63 \%$ reduction in the risk of progressive disease or death among patients in the lenalidomide group. The median time to progression was 39 months among patients in the lenalidomide group and 21 months among patients in the placebo group ( $\mathrm{P}<0.001)$ (Fig. S2a in the Supplementary Appendix). As of December 17, 2009 , a total of 13 of the 231 patients in the lenalidomide group (6\%) and 24 of the 229 patients in the placebo group (10\%) had died (hazard ratio, 0.52; 95\% CI, 0.26 to 1.02; twosided $\mathrm{P}=0.05$ ). The median overall survival had not been reached for either group (Fig. S2b in the Supplementary Appendix). The primary end point (time to progression) was met, and the study was unblinded so that patients in the placebo group could cross over to lenalidomide therapy. Of 128 eligible patients without disease progression in the placebo group, 86 received lenalidomide therapy. 
The median follow-up as of October 31, 2011, was 34 months. Eighty-six of the 231 patients in the lenalidomide group (37\%) as compared with 132 of the 229 patients in the placebo group (58\%) had disease progression or had died (hazard ratio, 0.48 ; 95\% CI, 0.36 to 0.63 ). The median time to progression was 46 months in the lenalidomide group and 27 months in the placebo group $(\mathrm{P}<0.001)$ (Fig. 1A). The 3-year rate of freedom from progression or death was 66\% (95\% CI, 59 to 73) among patients in the lenalidomide group and 39\% (95\% CI, 33 to 48) among patients in the placebo group. A total of 35 patients who received lenalidomide (15\%) and 53 patients who received placebo (23\%) died (two-sided $\mathrm{P}=0.03$ ). Thus, $85 \%$ of the patients in the lenalidomide group and $77 \%$ of the patients in the placebo group were alive at the time of the analysis. The rate of overall survival at 3 years was $88 \%$ (95\% CI, 84 to 93) among patients in the lenalidomide group and $80 \%$ (95\% CI, 74 to 86) among patients in the placebo group (hazard ratio, $0.62 ; 95 \%$ CI, 0.40 to 0.95 ) (Fig. 1B).

\section{TIME TO PROGRESSION ACCORDING TO STRATIFICATION AT RANDOMIZATION}

Time to progression according to the $\beta_{2}$-microglobulin level at registration (normal vs. elevated) is shown in Figure S2c in the Supplementary Appendix. Figure S2d in the Supplementary Appendix shows time to progression according to status with respect to prior induction therapy with thalidomide (yes vs. no), and Figure S2e in the Supplementary Appendix shows time to progression according to status with respect to prior induction therapy with lenalidomide (yes vs. no). Figure 2A shows a forest plot comparing the relative influence of stratification factors on time to progression. There was a trend toward a greater difference in time to progression with lenalidomide than with placebo for status with respect to lenalidomide induction therapy ( $\mathrm{P}=0.06$ for interaction).

\section{TIME TO PROGRESSION ACCORDING TO RESPONSE AT RANDOMIZATION}

Table 1 shows the responses of the patients to autologous hematopoietic stem-cell transplantation at randomization. The time to progression according to the response at randomization is shown for the analysis of data at the time of unblinding and for the later analysis in Figures S2f and S2g in the Supplementary Appendix, respectively. Figure 2A shows a forest plot comparing the relative influence of responses to induction therapy and transplantation and random assignment to lenalidomide or placebo on the time to progression in the two study groups. We cannot conclude that there was an interaction between remission status at randomization and maintenance therapy with respect to time to progression $(\mathrm{P}=0.38)$. However, lenalidomide maintenance therapy appeared to increase the time to progression in patients who did not have complete remission at day 100 after transplantation.

\section{OVERALL SURVIVAL ACCORDING TO STRATIFICATION AT RANDOMIZATION}

Figure S2h in the Supplementary Appendix shows overall survival according to the $\beta_{2^{-}}$ microglobulin level at randomization (normal vs. elevated). Figure S2i in the Supplementary Appendix shows overall survival according to status with respect to prior induction therapy with thalidomide (yes vs. no), and Figure S2j in the Supplementary Appendix shows overall survival according to status with respect to prior induction therapy with lenalidomide (yes vs. no). Overall survival did not differ significantly between the lenalidomide and placebo groups when they were stratified according to the $\beta_{2}$-microglobulin level and status with respect to prior thalidomide exposure. Figure $2 \mathrm{~B}$ shows a forest plot comparing the relative influence of responses to induction therapy and transplantation and random assignment to lenalidomide or placebo on overall survival. The data provide evidence that induction therapy with lenalidomide was associated with improved overall survival in the group that received lenalidomide maintenance therapy as compared with the placebo group $(\mathrm{P}=0.03)$. 


\section{ADVERSE EVENTS AND SECOND PRIMARY CANCERS}

Adverse events after randomization (up to February 2012) are summarized in Table 2 and in Tables S1 and S2 in the Supplementary Appendix. More grade 3 or 4 hematologic adverse events occurred in patients in the lenalidomide group than in the placebo group $(\mathrm{P}<0.001)$; in particular, more patients in the lenalidomide group had grade 3 or 4 neutropenia. There were no grade 5 hematologic adverse events. There were more grade 3 nonhematologic adverse events in the lenalidomide group than in the placebo group $(\mathrm{P}<0.001)$; there were no significant differences between the groups with respect to the numbers of grade 4 and grade 5 non-hematologic adverse events.

A total of 23 of 231 patients in the lenalidomide group discontinued therapy because of adverse events. Two of 143 patients in the placebo group who did not cross over to lenalidomide discontinued therapy because of adverse events, and 5 of 86 patients in the placebo group who crossed over to lenalidomide discontinued therapy because of adverse events (see Fig. S1a and S1b in the Supplementary Appendix).

After randomization, 8 new hematologic cancers and 10 solid-tumor cancers (excluding non-melanoma skin cancers) were diagnosed among the 231 patients in the lenalidomide group (3.5\% and $4.3 \%$, respectively). The corresponding numbers of new hematologic and solid-tumor cancers among the 229 patients in the placebo group were $1(0.4 \%)$ and 5 $(2.2 \%)$ (Table 3). The median time to the diagnosis of a hematologic cancer after randomization was 28 months (range, 12 to 46 ) in patients in the lenalidomide group, and the 1 hematologic cancer that occurred in a patient in the placebo group was diagnosed at 30 months. The median time to the diagnosis of a solid-tumor cancer after randomization was 15 months (range, 3 to 51) in the lenalidomide group and 21 months (range, 6 to 34 ) in the placebo group. Four of 10 patients with solid tumors restarted lenalidomide after surgery for their second primary cancer. One case of a second primary cancer (melanoma) was reported in a patient in the placebo group after crossover to lenalidomide.

\section{EVENT-FREE SURVIVAL AND CUMULATIVE INCIDENCE OF DISEASE PROGRESSION, SECOND PRIMARY CANCERS, AND DEATHS}

Event-free survival was a post hoc end point to assess the influence of second primary cancers on the observed time to progression and overall survival. Cases of nonmelanoma skin cancer (all local, without distant spread) were not classified as second primary cancers in the analysis. As of October 31, 2011, a total of 92 of the 231 patients in the lenalidomide group (40\%) as compared with 133 of the 229 patients in the placebo group (58\%) had progressive disease, had died, or had received a diagnosis of a second primary cancer $(\mathrm{P}<0.001)$. The estimated hazard ratio was 0.53 (95\% CI, 0.41 to 0.69 ), indicating a $47 \%$ reduction in risk among patients in the lenalidomide group. The median event-free survival was 43 months among patients in the lenalidomide group and 27 months among patients in the placebo group (Fig. S2k in the Supplementary Appendix). To further assess the causespecific risk profiles, we estimated the cumulative incidence risks of a second primary cancer, disease progression, and death, stratified according to group. The cumulative incidence of a second primary cancer was higher among patients in the lenalidomide group than among patients in the placebo group $(\mathrm{P}=0.008)$. The cumulative incidence of progressive disease and the cumulative incidence of death were higher among patients in the placebo group than among patients in the lenalidomide group $(\mathrm{P}<0.001$ and $\mathrm{P}=0.002$, respectively) (Fig. 3). 


\section{DISCUSSION}

Although a cure for multiple myeloma is still not possible in most patients, maintenance of a prolonged progression-free interval with minimal toxicity is an important goal in the management of this disease. The median overall survival among patients who required therapy before 1996 was approximately 3 years. ${ }^{23}$ In the era of new agents and autologous hematopoietic stem-cell transplantation, the median overall survival after transplantation is close to 8 years. ${ }^{23,24}$ In this study, $85 \%$ of patients in the lenalidomide group and $77 \%$ of patients in the placebo group were alive at a median follow-up of nearly 3 years.

Several strategies have been implemented to improve the response to primary therapy since it correlates with the outcome. ${ }^{25-27}$ Patients with multiple myeloma who have complete remission after primary therapy appear to have a longer time to progression, resulting in prolonged overall survival, although patients with a very good partial response (>90\% reduction in myeloma protein) may have excellent outcomes. ${ }^{25}$ Maintenance of disease control without clinically significant progression and dose-limiting toxic effects, as well as tolerability for the patient, may also translate into prolonged overall survival. Lenalidomide maintenance may increase the time to progression in patients who do not have complete remission after induction therapy and transplantation, thus generating outcomes similar to those for patients with complete remission. Although the response criteria of the International Myeloma Working Group were not used for all patients in this study, the results are consistent with those of previous studies with respect to response and outcome.

Diagnostic cytogenetic abnormalities in multiple myeloma have been associated with the outcome. ${ }^{28}$ Cytogenetic analysis was not required for enrollment in this study; however, a review of available data is ongoing. We expect this information to better define populations that would benefit most from lenalidomide maintenance therapy. Patients with disease progression before day 100 after autologous hematopoietic stem-cell transplantation (4\% of the patients who were registered in our study) were not eligible to undergo randomization. We cannot conclusively say whether induction regimens with multiple drugs could overcome progressive disease and whether this group of patients would benefit from maintenance therapy after transplantation.

Consolidation therapy after induction therapy and transplantation is one strategy that improves outcomes. Consolidation appears to be most effective in inducing complete remission in patients with residual disease. ${ }^{29,30}$ Consolidation therapy is more intensive than maintenance therapy, often with toxic effects. In this study, lenalidomide maintenance as a form of prolonged therapy, as compared with placebo, prolonged the time to progression and increased overall survival.

Despite its demonstrated efficacy, thalidomide maintenance therapy has been limited by neurotoxicity, with up to $75 \%$ of patients discontinuing maintenance therapy. ${ }^{7-12}$ Other studies have shown that lenalidomide and bortezomib used as maintenance therapy are better tolerated, with clinically significant efficacy for long-term maintenance after autologous hematopoietic stem-cell transplantation. ${ }^{31,32}$ In this issue of the Journal, Attal et al. ${ }^{33}$ also report a significantly prolonged time to disease progression with lenalidomide maintenance therapy after autologous hematopoietic stem-cell transplantation. A related article by Palumbo et al. ${ }^{34}$ describes a significantly prolonged time to disease progression with lenalidomide maintenance therapy after the use of low-dose induction therapy. These three studies show the usefulness of lenalidomide maintenance therapy for prolonging the time to disease progression in both patients who have undergone stem-cell transplantation and those who have not. The study reported by Attal et al. ${ }^{33}$ did not show an overall survival benefit, a finding that could be due to differences in induction (use or nonuse of 
lenalidomide-based induction therapy) and consolidation (use or nonuse of more alkylatorbased chemotherapy) before transplantation, the use of lenalidomide consolidation therapy in both groups after transplantation, the use of two transplantations in some patients, and the discontinuation of maintenance therapy. ${ }^{33}$ Longer follow-up and additional studies may clarify the different findings.

A major concern during maintenance therapy is toxicity that limits long-term use and the ability to receive future treatment after disease progression or that results in life-threatening disorders. Acute myeloid leukemia or the myelodysplastic syndrome has been reported in patients with multiple myeloma who did not undergo transplantation and were treated with melphalan. ${ }^{35,36}$ An observational bias is unlikely to explain these findings because of the rapid development of acute myeloid leukemia and, to a lesser extent, the my-elodysplastic syndrome. A recent report by the Swedish Cancer Registry described an increased incidence of these disorders in patients with multiple myeloma and monoclonal gammopathy of undetermined significance (MGUS) ${ }^{37}$ The finding that acute myeloid leukemia or the myelodysplastic syndrome occurs in untreated patients with MGUS suggests that these plasma-cell disorders are associated with a hematopoietic stem-cell or microenvironmental defect in addition to an effect of chemotherapy exposure. Multiple myeloma is also associated with solid-tumor cancers. ${ }^{38}$ In this study, the increase in second primary solidtumor cancers in the lenalidomide group was not associated with a specific tumor type, and the cause was uncertain. Close monitoring of blood counts, as indicated by the study guidelines, and standard screening for cancers are recommended.

In conclusion, this study suggests that lenalidomide maintenance therapy until disease progression is feasible for prolonged administration. The increase in time to progression led to early study unblinding, and despite the crossover, benefits with respect to progression and overall survival were seen in patients receiving lenalidomide maintenance therapy, especially those who had received lenalidomide-based induction therapy. It remains to be determined whether the incorporation of other new agents with lenalidomide will further increase the time to disease progression and overall survival.

\section{Supplementary Material}

Refer to Web version on PubMed Central for supplementary material.

\section{Acknowledgments}

The research was supported, in part, by grants from the National Cancer Institute and the National Heart, Lung, and Blood Institute.

\section{References}

1. Stewart AK, Richardson PG, San-Miguel JF. How I treat multiple myeloma in younger patients. Blood. 2009; 114:5436-43. [Erratum, Blood 2010;115:4006.]. [PubMed: 19861683]

2. Palumbo A, Anderson K. Multiple myeloma. N Engl J Med. 2011; 364:1046-60. [PubMed: 21410373]

3. Southwest Oncology Group Study. Remission maintenance therapy for multiple myeloma. Arch Intern Med. 1975; 135:147-52. [PubMed: 1111463]

4. Belch A, Shelley W, Bergsagel D, et al. A randomized trial of maintenance versus no maintenance melphalan and prednisone in responding multiple myeloma patients. Br J Cancer. 1988; 57:94-9. [PubMed: 3279997]

5. Fritz E, Ludwig H. Interferon-alpha treatment in multiple myeloma: meta-analysis of 30 randomised trials among 3948 patients. Ann Oncol. 2000; 11:1427-36. [PubMed: 11142483] 
6. Berenson JR, Crowley JJ, Grogan TM, et al. Maintenance therapy with alternate-day prednisone improves survival in multiple myeloma patients. Blood. 2002; 99:3163-8. [PubMed: 11964279]

7. Attal M, Harousseau JL, Leyvraz S, et al. Maintenance therapy with thalidomide improves survival in patients with multiple myeloma. Blood. 2006; 108:3289-94. [PubMed: 16873668]

8. Spencer A, Prince HM, Roberts AW, et al. Consolidation therapy with low-dose thalidomide and prednisolone prolongs the survival of multiple myeloma patients undergoing a single autologous stem-cell transplantation procedure. J Clin Oncol. 2009; 27:1788-93. [PubMed: 19273705]

9. Barlogie B, Pineda-Roman M, van Rhee F, et al. Thalidomide arm of Total Therapy 2 improves complete remission duration and survival in myeloma patients with metaphase cytogenetic abnormalities. Blood. 2008; 112:3115-21. [PubMed: 18492953]

10. Lokhorst HM, van der Holt B, Zweegman S, et al. A randomized phase 3 study on the effect of thalidomide combined with adriamycin, dexamethasone, and high-dose melphalan, followed by thalidomide maintenance in patients with multiple myeloma. Blood. 2010; 115:1113-20. [PubMed: 19880501]

11. Morgan GJ, Gregory WM, Davies FE, et al. The role of maintenance thalidomide therapy in multiple myeloma: MRC Myeloma IX results and meta-analysis. Blood. 2012; 119:7-15. [PubMed: 22021371]

12. Krishnan A, Pasquini MC, Logan B, et al. Autologous haemopoietic stem-cell transplantation followed by allogeneic or autologous haemopoietic stem-cell transplantation in patients with multiple myeloma (BMT CTN 0102): a phase 3 biological assignment trial. Lancet Oncol. 2011; 12:1195-203. [PubMed: 21962393]

13. Hideshima T, Mitsiades C, Tonon G, Richardson PG, Anderson KC. Understanding multiple myeloma pathogenesis in the bone marrow to identify new therapeutic targets. Nat Rev Cancer. 2007; 7:585-98. [PubMed: 17646864]

14. Richardson PG, Schlossman RL, Weller E, et al. Immunomodulatory drug CC-5013 overcomes drug resistance and is well tolerated in patients with relapsed multiple myeloma. Blood. 2002; 100:3063-7. [PubMed: 12384400]

15. Oken MM, Creech RH, Tormey DC, et al. Toxicity and response criteria of the Eastern Cooperative Oncology Group. Am J Clin Oncol. 1982; 5:649-55. [PubMed: 7165009]

16. Bladé J, Samson D, Reece D, et al. Criteria for evaluating disease response and progression in patients with multiple myeloma treated by high-dose therapy and haemopoietic stem cell transplantation. Br J Haematol. 1998; 102:1115-23. [PubMed: 9753033]

17. Durie BG, Harousseau JL, Miguel JS, et al. International uniform response criteria for multiple myeloma. Leukemia. 2006; 20:1467-73. [Errata, Leukemia 2006; 20:2220, 2007;21:1134.]. [PubMed: 16855634]

18. Kaplan EL, Meier P. Nonparametric estimation from incomplete observations. J Am Stat Assoc. 1958; 53:457-81.

19. Cox, DR.; Oakes, D. Analysis of survival data. London: Chapman \& Hall; 1984.

20. Kalbfleisch, JD.; Prentice, RL. The statistical analysis of failure time data. 2. Hoboken, NJ: John Wiley; 2002. p. 248-66.

21. Gray RJ. A class of K-sample tests for comparing the cumulative incidence of a competing risk. Ann Stat. 1988; 16:1141-54.

22. Fisher RA. The logic of inductive inference. J R Stat Soc [A]. 1935; 98:39-54.

23. Kumar SK, Rajkumar SV, Dispenzieri A, et al. Improved survival in multiple myeloma and the impact of novel therapies. Blood. 2008; 111:2516-20. [PubMed: 17975015]

24. Barlogie B, Attal M, Crowley J, et al. Long-term follow-up of autotransplantation trials for multiple myeloma: update of protocols conducted by the Intergroupe Francophone du Myelome, Southwest Oncology Group, and University of Arkansas for Medical Sciences. J Clin Oncol. 2010; 28:1209-14. [Erratum, J Clin Oncol 2010;28:3543.]. [PubMed: 20085933]

25. Harousseau JL, Avet-Loiseau H, Attal M, et al. Achievement of at least very good partial response is a simple and robust prognostic factor in patients with multiple myeloma treated with high-dose therapy: long-term analysis of the IFM 99-02 and 99-04 Trials. J Clin Oncol. 2009; 27:5720-6. [PubMed: 19826130] 
26. Martinez-Lopez J, Blade J, Mateos MV, et al. Long-term prognostic significance of response in multiple myeloma after stem cell transplantation. Blood. 2011; 118:529-34. [PubMed: 21482708]

27. Paiva B, Vidriales MB, Cerveró J, et al. Multiparameter flow cytometric remission is the most relevant prognostic factor for multiple myeloma patients who undergo autologous stem cell transplantation. Blood. 2008; 112:4017-23. [PubMed: 18669875]

28. Avet-Loiseau H, Attal M, Moreau P, et al. Genetic abnormalities and survival in multiple myeloma: the experience of the Intergroupe Francophone du Myélome. Blood. 2007; 109:3489_ 95. [PubMed: 17209057]

29. Ladetto M, Pagliano G, Ferrero S, et al. Major tumor shrinking and persistent molecular remissions after consolidation with bortezomib, thalidomide, and dexamethasone in patients with autografted myeloma. J Clin Oncol. 2010; 28:2077-84. [PubMed: 20308672]

30. Cavo M, Tacchetti P, Patriarca F, et al. Bortezomib with thalidomide plus dexamethasone compared with thalidomide plus dexamethasone as induction therapy before, and consolidation therapy after, double autologous stem-cell transplantation in newly diagnosed multiple myeloma: a randomised phase 3 study. Lancet. 2010; 376:2075-85. [Erratum, Lancet 2011; 378:1846.]. [PubMed: 21146205]

31. Palumbo A, Gay F, Falco P, et al. Bortezomib as induction before autologous transplantation, followed by lenalidomide as consolidation-maintenance in untreated multiple myeloma patients. $\mathrm{J}$ Clin Oncol. 2010; 28:800-7. [PubMed: 20048187]

32. Neben K, Lokhorst HM, Jauch A, et al. Administration of bortezomib before and after autologous stem cell transplantation improves outcome in multiple myeloma patients with deletion $17 \mathrm{p}$. Blood. 2012; 119:940-8. [PubMed: 22160383]

33. Attal M, Lauwers-Cances V, Marit G, et al. Lenalidomide maintenance after stem-cell transplantation for multiple myeloma. N Engl J Med. 2012; 366:1782-91. [PubMed: 22571202]

34. Palumbo A, Hajek R, Delforge M, et al. Continuous lenalidomide treatment for newly diagnosed multiple myeloma. N Engl J Med. 2012; 366:1759-69. [PubMed: 22571200]

35. Kyle RA, Pierre RV, Bayrd ED. Multiple myeloma and acute myelomonocytic leukemia. N Engl J Med. 1970; 283:1121-5. [PubMed: 5273282]

36. Andersen E, Videbaek A. Stem cell leukaemia in myelomatosis. Scand J Haematol. 1970; 7:201-7. [PubMed: 5270658]

37. Mailankody S, Pfeiffer RM, Kristinsson SY, et al. Risk of acute myeloid leukemia and myelodysplastic syndromes after multiple myeloma and its precursor disease (MGUS). Blood. 2011; 118:4086-92. [PubMed: 21795746]

38. Hasskarl J, Ihorst G, De Pasquale D, et al. Association of multiple myeloma with different neoplasms: systematic analysis in consecutive patients with myeloma. Leuk Lymphoma. 2011; 52:247-59. [PubMed: 21054148]

\section{APPENDIX}

The authors' affiliations are as follows: Blood and Marrow Transplant Program, Roswell Park Cancer Institute, Buffalo (P.L.M.), Hematology Oncology, State University of New York Health Science Center, Syracuse (T.G.), Memorial Sloan-Kettering Cancer Center (H.H., S.G., H.L.), and BMT, Mount Sinai Medical Center, New York (L.I.) - all in New York; the Department of Biostatistics and Bioinformatics (K.O.) and Cancer and Leukemia Group B (CALGB) Statistical Center (K.O., V.H., J.P., C.J., E.B., S.B.), Duke University, Durham, Wake Forest University, Winston-Salem (D.D.H., D.L.), and Department of Medicine, University of North Carolina, Chapel Hill (D.A.G., T.C.S.) - all in North Carolina; the Division of Hematology, Ohio State University Comprehensive Cancer Center, Columbus (C.C.H., S.M.D.); the Department of Medical Oncology, Jerome Lipper Multiple Myeloma Center, Dana-Farber Cancer Institute, Boston (P.G.R., R.S., K.C.A.); Myeloma Program, Abramson Cancer Center, University of Pennsylvania, Philadelphia (E.A.S.); the Department of Hematology and Oncology, University of Minnesota Medical Center, Minneapolis (D.J.W., B.M.); the Division of Oncology, Washington University School of Medicine in St. Louis, St. Louis (R.V.); Bone Marrow Transplant Program, University of 
Florida, Gainesville (J.S.M.); the Department of Hematology, University of Wisconsin, Madison (N.S.C.); the Department of Medicine, Medical College of Wisconsin, Center for International Blood and Marrow Transplant Research, Milwaukee (P.H., M.C.P., M.M.H.); the Department of Medicine, University of Chicago Hospitals (K.V.B., C.R.), and CALGB, University of Chicago (L.B., M.K., M.S.) - both in Chicago; Oregon Health and Science University, Portland (R.T.M.); BMT Group of Georgia, Atlanta (A.B.); the Department of Hematology and Oncology, University of California San Francisco, San Francisco (T.M., C.L.); and Stem Cell Transplant and Cellular Therapy, Unit 423, University of Texas M.D. Anderson Cancer Center, Houston (M.H.Q.). 


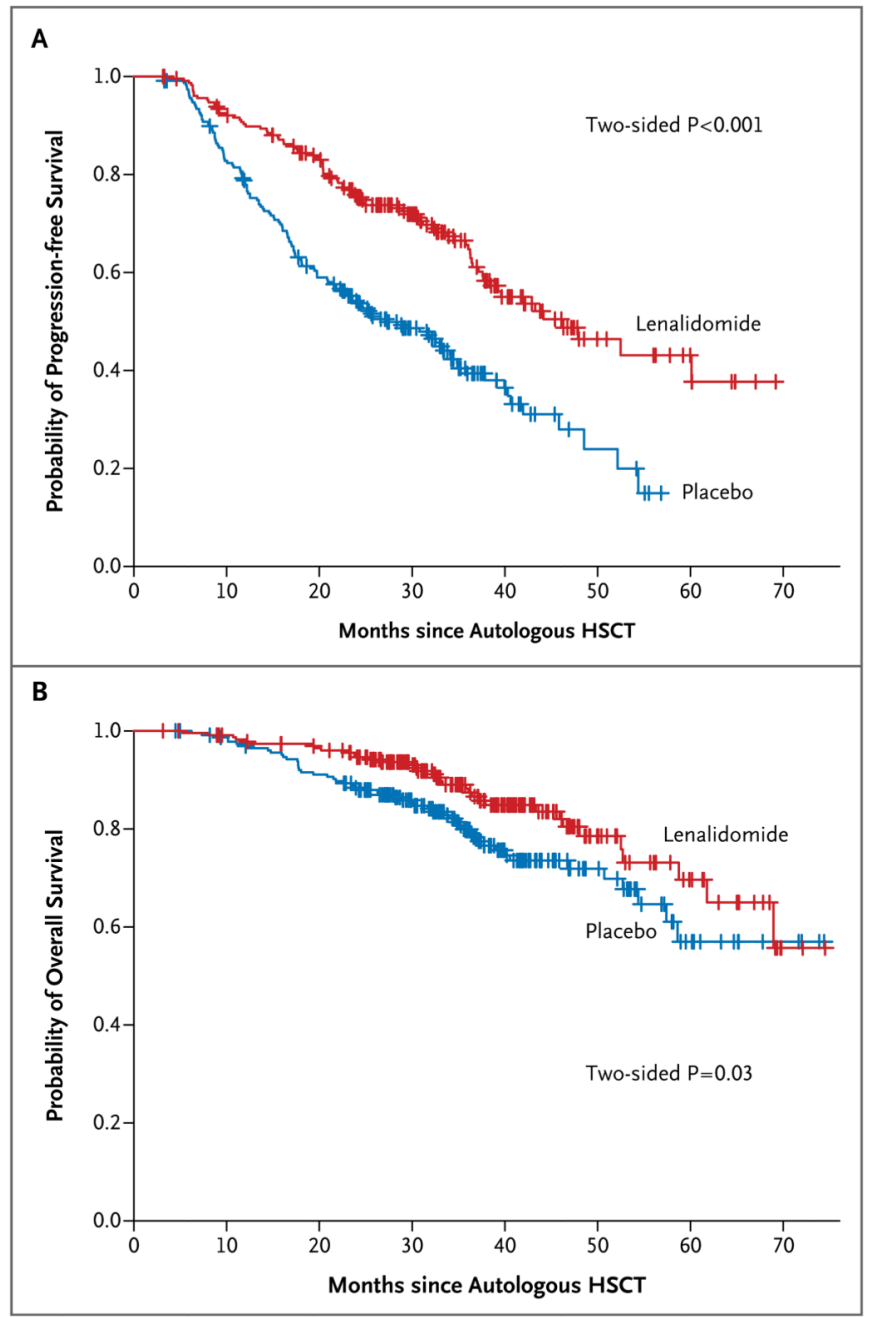

Figure 1. Kaplan-Meier Estimates of Progression-free and Overall Survival HSCT denotes hematopoietic stem-cell transplantation. 


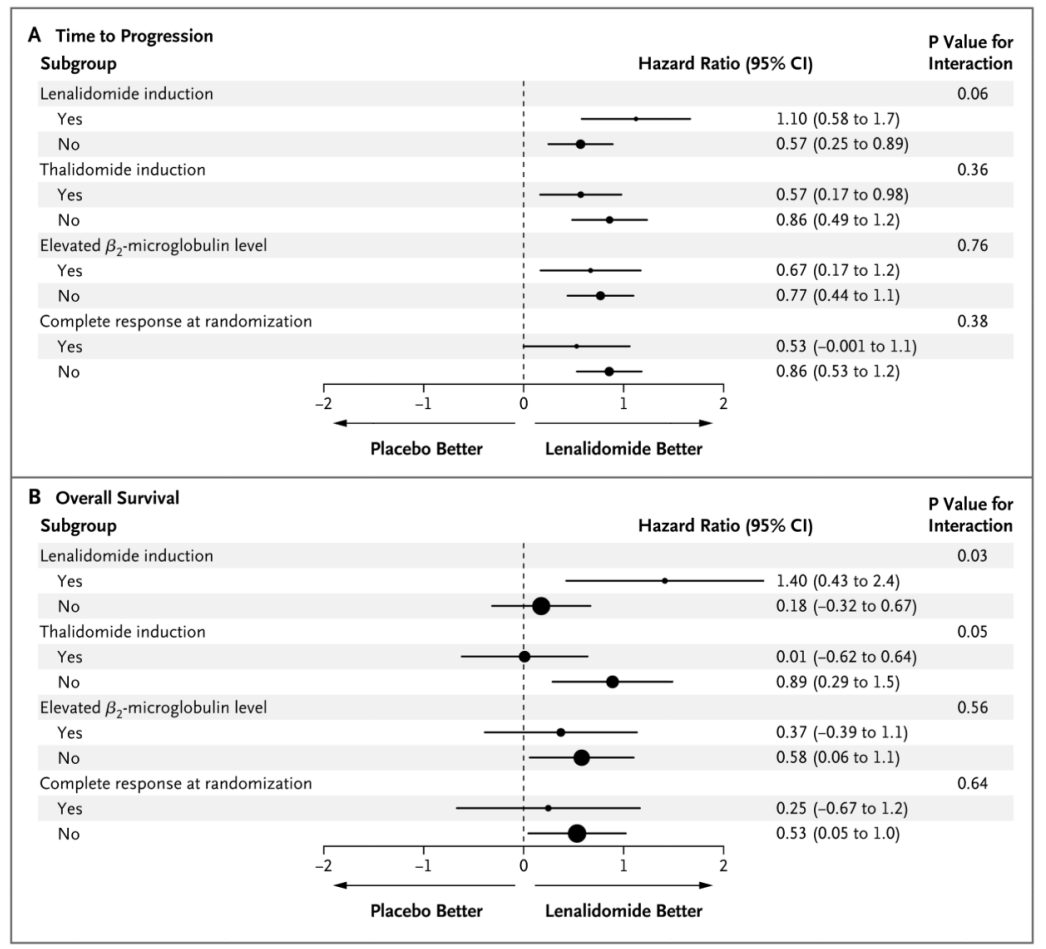

Figure 2. Forest Plot of Time to Progression and Overall Survival Hazard ratios from subgroup analyses of time to disease progression and overall survival in the randomized population are shown (on a natural-log scale). The radii of the circles are proportional to the inverse of the square of the standard error. 


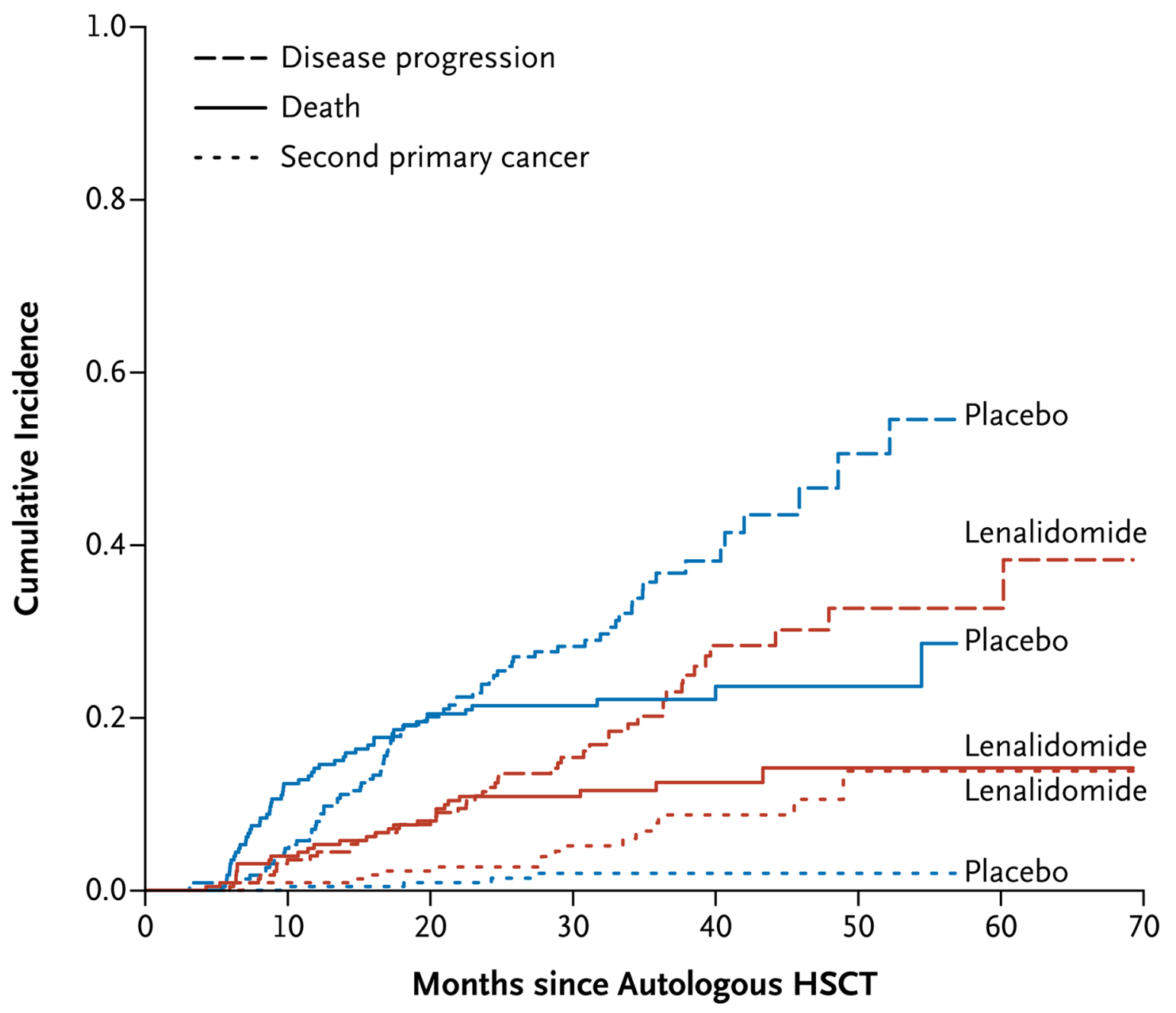

Figure 3. Cumulative Incidence of Second Primary Cancers, Disease Progression, and Death in the Lenalidomide and Placebo Groups after Randomization

The cumulative incidence risk of second primary cancers was greater in the lenalidomide group than in the placebo group $(\mathrm{P}=0.0008)$. The cumulative incidence risks of progressive disease and death were greater in the placebo group $(\mathrm{P}<0.001$ for progression and $\mathrm{P}=0.002$ for death). All $\mathrm{P}$ values are two-sided. 
Table 1

Characteristics of the Patients. *

\begin{tabular}{|c|c|c|c|}
\hline Characteristic & Lenalidomide $(\mathrm{N}=\mathbf{2 3 1}$ ) & Placebo $(N=229)$ & Total $(N=460)$ \\
\hline \multicolumn{4}{|l|}{ Age - yr } \\
\hline Median & 59 & 58 & 59 \\
\hline Range & $29-71$ & $40-71$ & $29-71$ \\
\hline Male sex — no. & 121 & 129 & 250 \\
\hline \multicolumn{4}{|c|}{$\beta_{2}$-microglobulin at registration - no. $(\%)$} \\
\hline$>2.5 \mathrm{mg} / \mathrm{liter}$ & $50(22)$ & $55(24)$ & $105(23)$ \\
\hline$\underline{2} .5 \mathrm{mg} / \mathrm{liter}$ & $170(74)$ & $163(71)$ & $333(72)$ \\
\hline Data missing & $11(5)$ & $11(5)$ & $22(5)$ \\
\hline \multicolumn{4}{|c|}{ Durie-Salmon stage at registration - no. $(\%)^{\dagger}$} \\
\hline I & $35(15)$ & $28(12)$ & $63(14)$ \\
\hline II & $71(31)$ & $59(26)$ & $130(28)$ \\
\hline III & $112(48)$ & $129(56)$ & $241(52)$ \\
\hline Data missing & $13(6)$ & $13(6)$ & $26(6)$ \\
\hline \multicolumn{4}{|l|}{ M component - no. $(\%)$} \\
\hline \multicolumn{4}{|l|}{ Serum } \\
\hline IgG kappa & $70(30)$ & $76(33)$ & $146(32)$ \\
\hline IgG lambda & $43(19)$ & $31(14)$ & $74(16)$ \\
\hline IgA kappa & $21(9)$ & $20(9)$ & $41(9)$ \\
\hline IgA lambda & $13(6)$ & $13(6)$ & $26(6)$ \\
\hline IgM kappa & $2(1)$ & $1(<1)$ & $3(1)$ \\
\hline IgM lambda & 0 & $1(<1)$ & $1(<1)$ \\
\hline \multicolumn{4}{|l|}{ Urine } \\
\hline Kappa light chain only & $13(6)$ & $12(5)$ & $24(5)$ \\
\hline Lambda light chain only & $4(2)$ & $10(4)$ & $14(3)$ \\
\hline Data missing & $35(15)$ & $41(18)$ & $76(17)$ \\
\hline Nonsecretory myeloma - no. $(\%)$ & $30(13)$ & $24(10)$ & $54(12)$ \\
\hline \multicolumn{4}{|l|}{ Serum calcium at registration $-\mathrm{mg} / \mathrm{dl}$} \\
\hline Median & 9.1 & 9.1 & 9.1 \\
\hline Range & $7.2-12.8$ & $3.1-10.8$ & $7.2-12.8$ \\
\hline \multicolumn{4}{|l|}{ Serum albumin at registration $-\mathrm{g} / \mathrm{dl}$} \\
\hline Median & 4.0 & 3.9 & 4.0 \\
\hline Range & $1.4-4.9$ & $2.9-5.0$ & $1.4-5.0$ \\
\hline \multicolumn{4}{|c|}{ Serum creatinine at registration $-\mathrm{mg} / \mathrm{dl}$} \\
\hline Median & 0.9 & 0.9 & 0.9 \\
\hline Range & $0.4-1.9$ & $0.5-2.2$ & $0.4-2.2$ \\
\hline
\end{tabular}




\begin{tabular}{|c|c|c|c|}
\hline Characteristic & Lenalidomide $(\mathrm{N}=\mathbf{2 3 1})$ & Placebo $(N=229)$ & Total $(N=460)$ \\
\hline I & $177(77)$ & $170(74)$ & $347(75)$ \\
\hline II & $11(5)$ & $16(7)$ & $26(6)$ \\
\hline III & $4(2)$ & $3(1)$ & $7(2)$ \\
\hline Data missing & $39(17)$ & $40(17)$ & $79(17)$ \\
\hline \multicolumn{4}{|l|}{ Induction regimen - no. } \\
\hline Any use of bortezomib & 98 & 91 & $189(41)$ \\
\hline Any use of lenalidomide & 79 & 81 & $160(35)$ \\
\hline Any use of thalidomide & 102 & 103 & $205(45)$ \\
\hline Bortezomib-lenalidomide ${ }^{* t}$ & 20 & 21 & $41(9)$ \\
\hline Bortezomib-thalidomide $f$ & 33 & 27 & $60(13)$ \\
\hline Bortezomib without lenalidomide or thalidomide & 43 & 40 & $83(18)$ \\
\hline Bortezomib with glucocorticoids, without lenalidomide or thalidomide & 40 & 32 & $72(16)$ \\
\hline Bortezomib with lenalidomide and thalidomide & 2 & 3 & $5(1)$ \\
\hline Lenalidomide without bortezomib & 57 & 57 & $114(25)$ \\
\hline Thalidomide without bortezomib & 67 & 72 & $139(30)$ \\
\hline Lenalidomide-glucocorticoids without bortezomib & 56 & 56 & $112(24)$ \\
\hline Thalidomide-glucocorticoids without bortezomib & 65 & 72 & $137(30)$ \\
\hline $\begin{array}{l}\text { Other induction regimen without bortezomib, lenalidomide, or } \\
\text { thalidomide }\end{array}$ & 15 & 13 & $28(6)$ \\
\hline Other induction regimen not determined & 0 & 1 & $1(<1)$ \\
\hline \multicolumn{4}{|l|}{ Response to autologous HSCT at day $100-$ no. (\%) } \\
\hline Complete response & $67(29)$ & $79(34)$ & $146(32)$ \\
\hline Partial response & $115(50)$ & $109(48)$ & $224(49)$ \\
\hline Marginal response & $11(5)$ & $5(2)$ & $16(3)$ \\
\hline Stable disease & $38(16)$ & $32(14)$ & $70(15)$ \\
\hline Progressive disease & 0 & $3(1)$ & $3(1)$ \\
\hline Data missing & 0 & $1(<1)$ & $1(<1)$ \\
\hline Mean time from autologous HSCT to randomization - mo & 3.3 & 3.3 & \\
\hline
\end{tabular}

"The first patient was enrolled in April 2005, and the study was closed to enrollment in July 2009. To convert the values for serum $\beta_{2}$ microglobulin to nanomoles per liter, multiply by 84.75 . To convert the values for calcium to millimoles per liter, multiply by 0.250 . To convert the values for creatinine to micromoles per liter, multiply by 88.4. There were no significant differences $(\mathrm{P}<0.05)$ between the lenalidomide and placebo groups with respect to baseline characteristics. There was an overlap in the induction regimens, so percentages may sum to more than $100 \%$. HSCT denotes hematopoietic stem-cell transplantation, and ISS International Staging System.

${ }^{\dagger}$ Higher stages indicate more severe disease. The staging criteria are defined in the Supplementary Appendix.

Fatients who received this regimen received at least these two drugs. 


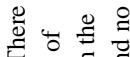

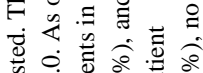

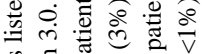

음

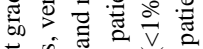

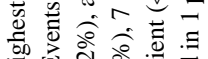

西

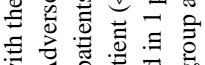

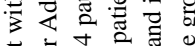

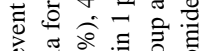

o $\overline{0}$ b

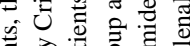

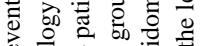

0

등

क ส

政

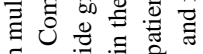

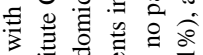

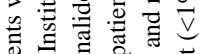

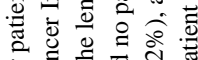

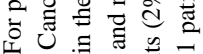

可高客高

$\Rightarrow$ 焉

ป气

กิ่

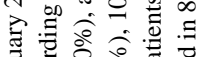

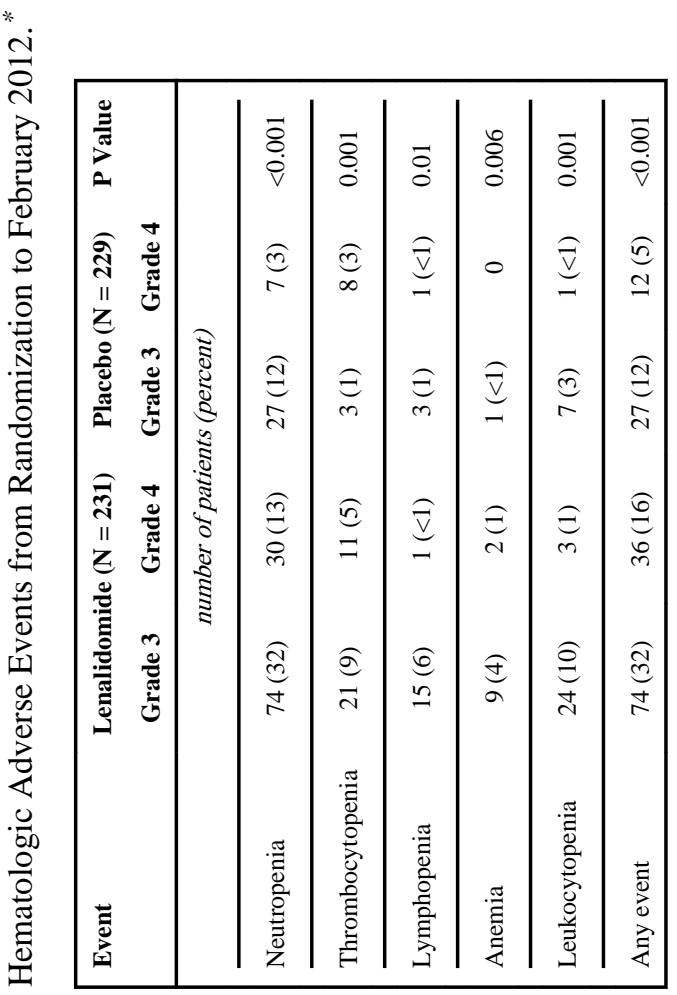

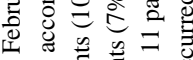

을

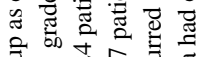

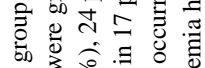

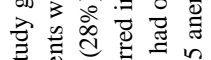

की

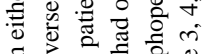

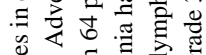

自园的

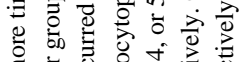

可

范势

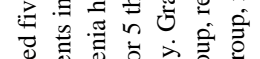

要

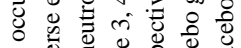

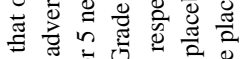

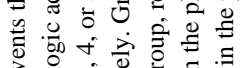

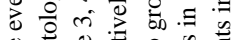

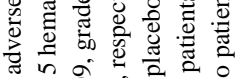

응 ठे

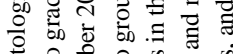

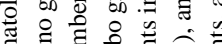


Table 3

Second Primary Cancers from Randomization to February 2012.

\begin{tabular}{|c|c|c|}
\hline Second Cancer & Lenalidomide $(\mathrm{N}=\mathbf{2 3 1})$ & Placebo $(N=229)$ \\
\hline & \multicolumn{2}{|c|}{ number of patients } \\
\hline \multicolumn{3}{|l|}{ Hematologic cancers * } \\
\hline Acute lymphoblastic leukemia & 1 & 0 \\
\hline Acute myeloid leukemia & 5 & 0 \\
\hline Hodgkin's lymphoma & 1 & 0 \\
\hline Myelodysplastic syndrome & 1 & 0 \\
\hline Non-Hodgkin's lymphoma & 0 & 1 \\
\hline Total & 8 & 1 \\
\hline \multicolumn{3}{|l|}{ Solid-tumor cancers } \\
\hline Breast cancer & 3 & 0 \\
\hline Carcinoid tumor & 0 & 1 \\
\hline Central nervous system cancer & 1 & 0 \\
\hline Gastrointestinal cancer & 2 & 1 \\
\hline Gynecologic cancer & 1 & 1 \\
\hline Malignant melanoma & 1 & 2 \\
\hline Prostate cancer & 1 & 0 \\
\hline Thyroid cancer & 1 & 0 \\
\hline Total & 10 & 5 \\
\hline Basal-cell carcinoma & 2 & 1 \\
\hline Squamous-cell carcinoma & 2 & 2 \\
\hline
\end{tabular}

Four of the eight patients with a hematologic cancer in the lenalidomide group received induction therapy with an anthracycline. One solid-tumor cancer occurred in a patient with breast cancer in the lenalidomide group. This patient had primary breast cancer 26 years before presentation with metastatic disease. It is not known whether this breast cancer was a new primary cancer or a recurrence of the original breast cancer. Two cases of solid tumors (one in the lenalidomide group and one in the placebo group) occurred after disease progression. These cases were not included in the analysis of second primary cancers, since they occurred after disease progression and then further therapy. 